Conclusions There was increased incidence and severity of DKA during the study period in 2020.Our study did not provide evidence that the COVID-19 pandemic is leading to a marked increase in incidence of pediatric type 1 diabetes.

\section{Quality Improvement and Patient Safety}

\section{A MOMENT FOR CHANGE. ADDRESSING PARENTAL SMOKING IN THE PAEDIATRIC EMERGENCY DEPARTMENT}

Yvette Redpath. Barking Havering and Redbridge NHS Trust

\subsection{6/archdischild-2021-rcpch.48}

Background Everyday in A+E children are attending with serious and life threatening breathing problems which we know are adversely affected by third hand smoking within the home.

In not addressing smoking with parents during their time in $\mathrm{A}+\mathrm{E}$, we fail to assist them in making a positive change for the health of their children. Using the biopsychosocial model, the impact on the child of their respiratory illness is wide ranging and far reaching.

Objectives

1. All respiratory cases presenting through $\mathrm{A}+\mathrm{E}$ to have $\mathrm{a}$ smoking status asked and documented

2. For smoking cessation advice to be given to every case for which a positive smoking status is recorded

Methods

Fishbone I used a fishbone diagram to explore the potential causes of poor smoking status history taking, and to establish potential change ideas

Main causes identified

1. Doctors forgetting to ask for smoking status

2. Fear of confrontation when asking smoking status

3. Lack of confidence in how to deliver smoking cessation advice

4. Doctor's workload pressures

I then used the Plan Do Study Act (PDSA) Cycle to implement change ideas

PDSA Cycle 1

CAUSES ADDRESSED

doctors forgetting to ask smoking status

Change Ideas:

1) Prompt sticker in clerking booklet

PDSA Cycle 2

\section{CAUSES ADDRESSED}

doctors forgetting to ask smoking status

lack of confidence in delivering smoking cessation advice

Change Ideas:

1. Prompt sticker in clerking booklet

2. Leaflet on smoking cessation and the harmful effects of third hand smoking

Data collection following change ideas

The change ideas were implemented, and data collected on what percentage of paediatric respiratory cases presenting to A $+\mathrm{E}$ had parental/carer smoking status documented, and of these what percentage were given smoking cessation advice following a positive smoking status.

\section{Results}

PDSA Cycle Percentage Improvements

Following PDSA Cycle 1 there was an increase from $35 \%$ to $71 \%$ of smoking status being documented and from $0 \%$ to $83 \%$ of the positive smoking status cases were given smoking cessation advice. Following PDSA Cycle 2 the status and advice given were $50 \%$ and $66 \%$ respectively.

\begin{tabular}{llll}
\hline & Pre-intervention & PDSA Cycle 1 & PDSA Cycle 2 \\
\hline Smoking status documented? & $35 \%$ & $71 \%$ & $50 \%$ \\
Smoking cessation advice given? & $0 \%$ & $83 \%$ & $66 \%$ \\
\hline
\end{tabular}

Conclusions Cycle 1 Change Idea achieved our aim; to improve the frequency of smoking status being asked and documented.

Cycle 2 Change Idea failed to achieve our aim. Smoking cessation advice is still not offered in the A+E setting for those with a positive smoking status.

Using a prompt sticker in the clerking booklet helped to improve the frequency of smoking status being asked and documented, however it still seems that doctors are not providing smoking cessation advice, even when a leaflet handout is provided. It is well known that third-hand smoking exacerbates childhood respiratory illness, increasing the frequency and severity of presentations. In not providing support and encouragement to parents who smoke in the home, we are failing to help them implement positive change for the health of their child. Further work to assist doctors in giving smoking cessation advice in the $\mathrm{A}+\mathrm{E}$ setting is needed.

\section{British Association of Perinatal Medicine and Neonatal Society}

\section{1 'JOURNEY OF ACCEPTANCE': A QUALITATIVE STUDY EXPLORING PASTEURISED DONOR HUMAN MILK USAGE AND ITS IMPACT ON MATERNAL EMOTIONAL WELLBEING}

${ }^{1}$ Hui Fang Loh, ${ }^{1}$ Jiayu Rachel Lee, ${ }^{1}$ Andrea Ruwen Tan, ${ }^{2}$ Xin Lei Goh, ${ }^{2}$ Yi Fen Low, ${ }^{1}$ Yvonne Peng Mei Ng. 'National University Hospital; ${ }^{2}$ National University of Singapore

\subsection{6/archdischild-2021-rcpch.49}

Background Human milk is the ideal food for all babies, especially vulnerable infants who are sick and premature. When mothers are unable to provide their babies with adequate breast milk, donor human milk is recommended to improve the infants' health outcomes. Singapore's first donor human milk (DHM) bank was established in August 2017 to provide pasteurised human donor milk to eligible babies, who are primarily premature infants below 35 weeks' gestational age. To date, more than 1963 babies have received DHM. However, there is currently limited knowledge about maternal experiences of DHM usage in Singapore.

Objectives This study aimed to explore Singaporean mothers' perception of DHM, examine the impact of DHM usage on maternal emotional wellbeing, and help inform clinicians to better support mothers of preterm infants in their breastfeeding journey. 
Methods Ethics approval was obtained from the National Healthcare Group Domain Specific Review Board (NHG DSRB). Convenience sampling was done to recruit mothers of premature babies hospitalised in the level II or level III Neonatal Intensive Care Unit, who had received donor human milk. In-depth semi structured Interviews were audiorecorded and transcribed verbatim. Transcripts were analysed using Braun and Clarke's six-step process of thematic analysis.

Results Seventeen mothers participated in this study. Many participants described their experience with DHM as a 'journey of acceptance'. Three supporting themes which illustrate this 'journey' include: (1) 'Resistance to receiving somebody else's milk,' (2) 'Recognising baby's needs and maternal limitations,' and (3) 'Embracing the benefits of donor milk'. Though not all mothers experienced the above in a chronological progression, many described a process of overcoming initial hesitation to eventual acceptance of feeding their child with another person's milk. Majority of mothers were initially unwilling to give DHM to their baby, citing concerns over the safety and quality of donor milk, as well as an innate responsibility to feed their own child. Feelings of sadness, disappointment, helplessness, guilt and self-blame set in when many realised that they were unable to meet their baby's demand for milk. For some Muslim mothers, such negative emotions were further aggravated as they considered their baby's health needs against implications of receiving DHM due to their Islamic faith. Nevertheless, all participants appreciated the benefits of donor human milk, often crediting their baby's growth and development to the combined effect of their own breast milk and DHM. Mothers who readily accepted DHM for their infants tended to be more open-minded and had greater self-awareness of their own limitations and their infant's needs.

Conclusions The experiences of these mothers reflect the spectrum of complex emotions faced when accepting an alternative source of human milk for their prematurely born infants. Healthcare practitioners should acknowledge such emotions in a culturally sensitive manner when sharing information or seeking parental consent to utilise donor milk for premature and sick babies.

\section{British Association of General Paediatrics}

\section{REVIEW OF A VIRTUAL WORK EXPERIENCE, USED TO SUPPORT SCHOOL-AGED STUDENTS DURING THE COVID-19 PANDEMIC}

${ }^{1}$ Emma Bailey, ${ }^{2}$ Robert Bailey, ${ }^{3}$ Prabu Rajendran, ${ }^{3}$ Sabine Hazell, ${ }^{3}$ Mhairi Emery. 'Lister Hospital: ${ }^{2}$ Caterham School; ${ }^{3}$ Bedfordshire NHS Foundation Trust

\subsection{6/archdischild-2021-rcpch.50}

Background The COVID-19 pandemic has had a huge impact on education, mental health and morale of school-aged students. Schools have been closed for months on end, meaning many have been left to ponder future careers with little or no help. Additionally, those considering a career within the NHS have been presented with press reports revealing how difficult working within this area can sometimes be but had little or no opportunity to visit or speak to professionals working in the roles. Thus, by holding a Virtual Work Experience for students who are interested in a career within the NHS we aimed to reduce anxieties and boost knowledge.

Objectives Our aims were to improve students' knowledge of what it is like to work within the NHS, as well as give insights into the multiple different career options that are available. In addition, we hoped the session would give students information to help them to go on and be successful in their applications and interviews.

In the long term, we hope this project will help the NHS and its' patients as we will have more informed young people joining the different professions.

Methods In partnership with the School Volunteering Team at Bedfordshire Hospitals NHS Foundation Trust, we invited school-aged students who were interested in exploring a career within the NHS to attend the session. We invited representatives from the Clinical (Doctor/Nursing student/Paramedic/GP nurse etc), Administrative and Management team along with Allied health professionals from both the Hospital and Community set-up to give presentations or video insights into their roles. Students were asked to complete a questionnaire pre and post-attending to help us evaluate what was useful and the effect the session had.

Results In total 26 students attended the day, 15 filled in the questionnaire pre-attending, and 14 post. $57 \%$ of students had done no medical work experience prior to this session, and of those who had, a third had only done virtual sessions.

From the questionnaires we found 12/14 students felt more confident in their decision to work in the NHS following the session, with the remaining 2/14 feeling no different. Additionally, we found $92 \%$ of students planned to use this event in their UCAS, personal statement and/or interview questions. Conclusions Young people have been hugely affected by the COVID-19 pandemic; they've been kept away from school, their peers and also missed opportunities for learning about future careers. Additionally, the current COVID-19 pandemic has placed the NHS in prime public view, revealing the hardships that often go along with the work.

Thus, by holding this event we hoped to allay fears, provide information and allow students to ask questions about entering into a profession within the NHS. The event's feedback was very positive, so we are now going on to hold a larger event in March 2021; inviting more students to attend and including more specialties

Therefore, by helping these young people at a time when access to services is so limited, we can help to continue to encourage them into careers within the NHS.

\section{George Still Forum: ADHD Disorders (ePoster presentations only)}

\section{QUALITY OF LIFE IN CHILDREN WITH ADHD - A LOCAL REVIEW}

${ }^{1}$ Nicole McGrath, ${ }^{2}$ Sunil Bhide, ${ }^{1}$ Nishadi Abeyweera. ${ }^{1}$ Kent community health NHS trust; ${ }^{2}$ Kent community health NHS foundation trust

\subsection{6/archdischild-2021-rcpch.51}

Background Quality of life (QoL) is an important consideration for children with long-term neurodevelopmental 